\title{
A Fuzzy Logic Inference System for Testing Lithium-ion Battery State of Charge
}

\author{
Chi Zhang ${ }^{1, \text { a }}$ * \\ ${ }^{1}$ College of Artificial Intelligence, National University of Defense Technology, Changsha, China \\ a chizhang0127@gmail.com \\ *corresponding author
}

Keywords: Lithium ion Battery, State of Charge, Voltage Recovery, Fuzzy Logic Inference System

\begin{abstract}
In this research the charge and discharge tests have been adopted as battery test methods. It has been considered that combining these methods with a fuzzy logic reference system (FIS) can be accurate and efficient in analyzing complex electrochemical processes, such as testing state of charge (SOC) for a lithium-ion battery (LIB). All the work have been carried out in Matlab/Simulink, including battery modelling, discharge testing, and fuzzy logic analysis. In this paper the approach combined FIS with discharge testing was proved to be of good accuracy, with the maximum relative error of SOC under $4 \%$, and it was found that the relative error increases as the tested SOC rises.
\end{abstract}

\section{Introduction}

Lithium-based battery has become the best choice for the consumer electronics market due to its advantages in being maintenance-free and dependable, and its small size and long runtime, which perfectly fits the demands present day of electronic devices. The report assessed the global lithiumion battery market generated revenues of \$3.1bn in 2017 [1]. The application for LIB covers from popular devices such as mobile phones to specialty applications, such as military and aerospace. In these special applications, the accurate indicator for battery SOC (state of charge) is vital in the whole process of important military or aerospace missions. In addition, the battery working environments make a difference to SOC due to temperature changes. Thus significance is attached to finding a reliable method to measure SOC.

SOC has become a current research topic in the battery-power applications [2]. Lots of efforts have been made on this field. Some researchers employed a method based on battery testing involving a fuzzy logic system for the calculation of SOC [3]-[10]. Fuzzy logic provides a possibility for us to make quick indications on the SOC values without monitoring the battery in the whole lifespan [2].

In this paper, we explored the methods to make indication on the SOC and proposed a quick battery SOC test system based on FIS in Simulink. The battery test includes charge and discharge tests. The charge and discharge test involved applying constant voltage or current and impulse current to the battery model and observing and comparing the curves of voltage dropping and rising continuously, or curves presenting a steep rise/drop in voltage and a gradual voltage recovery process back to the steady voltage value. With the curve datum, a fuzzy logic inference system which analyzes the battery SOC was able to be built.

\section{Theory}

\subsection{SOC}

Typically the criteria used to judge the battery state is SOC (state of charge), which can be derived from different parameters: internal impedance, capacity, voltage, self-discharge, charge acceptance, number of charge-discharge cycles [4]. From a capacity perspective, SOC can be defined as: 


$$
\mathrm{SOC}=\operatorname{SOC}_{0}+\frac{1}{C_{N}} \int_{0}^{t} i(\tau) d \tau
$$

Where $C_{N}$ is the nominal capacity, $S O C_{0}$ is the initial SOC, $i(\tau)$ is the charge current.It can be deduced from above that SOC is $100 \%$ when it is fully charged.

From a standardization draft which was proposed by IEEE in April 1992, the conventional method of measuring SOC should be transferred from a float voltage test to an impedance or admittance test as impedance is a more efficient and exact method to measure SOC [5].While it is not convenient to calculate SOC by online monitoring the battery voltage according to the form.(1), so a more efficient approach through battery test was brought up by many researchers.

\subsection{Battery testing system based on fuzzy logic}

The term fuzzy logic was introduced by Lotfi Zadeh in 1965 in his proposal of fuzzy set theory [6]. Fuzzy logic has wide applications from control theory to artificial intelligence, and it is always a considerable method to solve complicated nonlinear problems, and there are previous experiments shown that it is a reliable, robust and code efficient method to estimate SOC [7]-[11].

A normal FIS works like a black box consisting of four basic parts:

1) A rule base describing the relationship between input and output variables;

2) A data base that defines the membership functions for the input and, in the case of Mamdani modelling, output variables;

3) A reasoning mechanism that performs the inference procedure;

4) A defuzzification block that transforms the fuzzy output sets to a real valued output [3].

A battery testing system based on fuzzy logic can be seen as a system to obtain the evaluation of battery SOC from input parameters such as voltages, current and impedance, based on the rules built from previous experience datum. Among the previous work [7]-[11], the selection of different types of battery test is diverse. As for DC test, discharge and charge tests are common approaches adopted to evaluate battery performance, as charge and discharge curves are relatively easier to obtain. DC tests could fall into two categories. One uses a large constant discharge voltage or charge current to make the voltage drop or rise continuously, then transfer to using a small discharge current or charge voltage to make the battery fully discharged or charged [7]. Another one uses the impulse signal to stimulate the battery, resulting in a steep rise/drop in voltage values and a gradual voltage recovery process back to the steady voltage value [8]. Comparing these two tests, the former one could have more stable results, and the curves corresponding to different SOC should have different inflection point values respectively, which could be the inputs for the FIS, because the shapes of the curves are similar. For the latter one, the curves of impulse tests contain rich details as the stimulate source is steep and transient thus it causes complicated response signal containing lots of frequency components, so we can get more information in the frequency domain. In system analysis theory, the unit impulse response can utilized to describe the transform function of the system.

\section{Methodology}

The method adopted is to build a SOC measurement system in Simulink through a simple test done to a battery model. In the following content firstly we give description on the processes of building a battery model in Simulink, then making measurements on voltage recovery test, and finally building a fuzzy logic inference system.

\subsection{Battery model}

The battery model to be built should simulate the dynamic characteristics of voltage, current, impedance and temperature well. The battery model based on resistance equivalent circuit has 1RC model, 2RC model and 3RC model, the more (RC) elements there are, the more electrochemical processes happening inside battery can be simulated. Some battery models reflect the temperature influence on the battery performance while others cannot. The default battery cell model in MATLAB/Simulink SimPowerSystems library cannot reflect the influence temperature and cycle 
times have on battery performance [12]. It is necessary to build a battery model which can response to the change of environment.

The battery model selected here is based on the 2RC model. Every electronic element in the model is built from look-up tables and the needed experimental data can be referred from MathWorks website.

Figure. 1(a) is the inner structure of the battery model, composed of a voltage source look-up table, 3 resistance look-up tables and 2 capacity look-up tables, and also a thermal model to monitor the temperature of the battery model. These dependent subsystems work together as a whole battery model. Figure. 1(b) displays the working conditions of battery test. It is assumed that cooling is primarily via convection, and that heating is primarily from internal resistance.
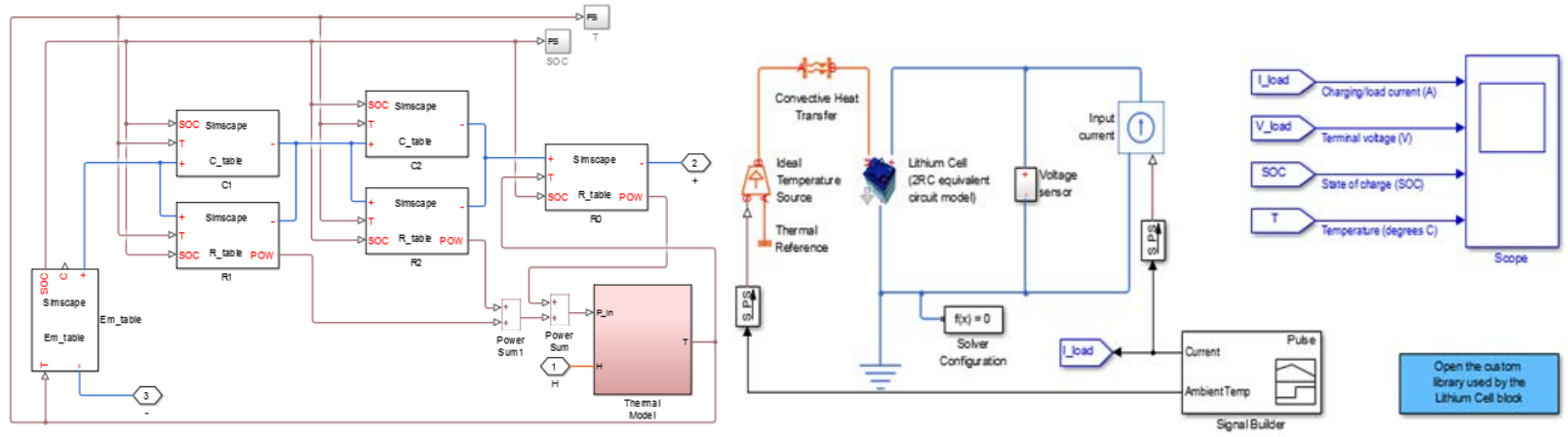

Figure 1 The battery model based on 2(RC) equivalent circuit in Simulink

\subsection{Voltage recovery}

The voltage recovery test is carried out on the battery model built above. The range of the current should be less than 2A as the peak load current for LIB is 2A [2]. The initial SOC value of the battery is set $80 \%$, and the ambient temperature is set at $20^{\circ} \mathrm{C}$. The first step is to apply a positive charge current impulse to the battery model to charge it till SOC reaches $100 \%$.

Later the load current applied to battery model comprises periodically discharging 2A pulses last for 750 s. During the 750 s discharge period, SOC will be reduced by $10 \%$. As the impulse time increases, the available capacity decreases, and so does the SOC.

The datum of the voltage recovery response after the removal of the current depression are collected and as well as the inner temperature of battery model. All the results are given below.

\subsection{Fuzzy logic inference system based on the discharge process}

The fuzzy logic inference system was built using the Fuzzy Logic Tool Box in MATLAB. There are three things to be solved. The selection of inference system, the input data space, and the membership function.

The Mamdani inference system and gauss membership function are chosen for the two inputs and one output. The choice of input data can be a key factor for accuracy and efficiency. These data are the expertise utilized to create a rule base describing the relationship between inputs and outputs. It is better to choose the parameters having a one-to- one corresponding relationship with SOC so more information can be reflected.

As shown by the Figure.2, the discharge process includes the constant discharge process in Figure. 2(a) and the voltage recovery process in Figure. 2(b). Input1 is chosen as the time when the battery is fully discharged (called peak time in Table. 1) from the discharge curves in Figure. 2(a) (only SOC is from100 to50 are shown) and input2 is chosen from Figure. 2 (b) as the steady state voltage value (called steady voltage in Table. 1) after each single discharge impulse. Table 1 below lists these extracted data.

Table 1 Input and Output datum for FIS

\begin{tabular}{|c|c|c|c|}
\hline No. & SOC (\%) & Peak time (s) & Steady voltage(v) \\
\hline 1 & 100 & 33 & 3.525 \\
\hline 2 & 90 & 568.7 & 3.384 \\
\hline
\end{tabular}




\begin{tabular}{|c|c|c|c|}
\hline 3 & 80 & 1289 & 3.352 \\
\hline 4 & 70 & 2009 & 3.343 \\
\hline 5 & 60 & 2729 & 3.331 \\
\hline 6 & 50 & 3449 & 3.323 \\
\hline
\end{tabular}

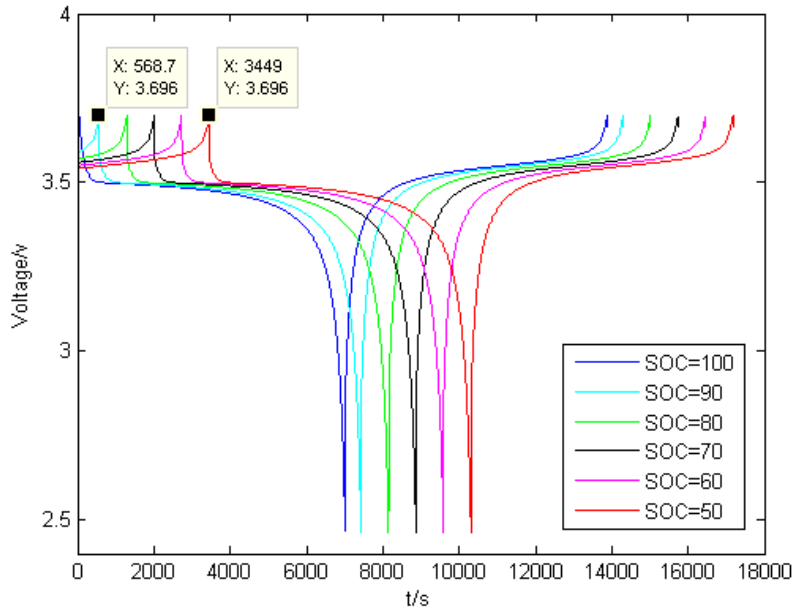

(a)

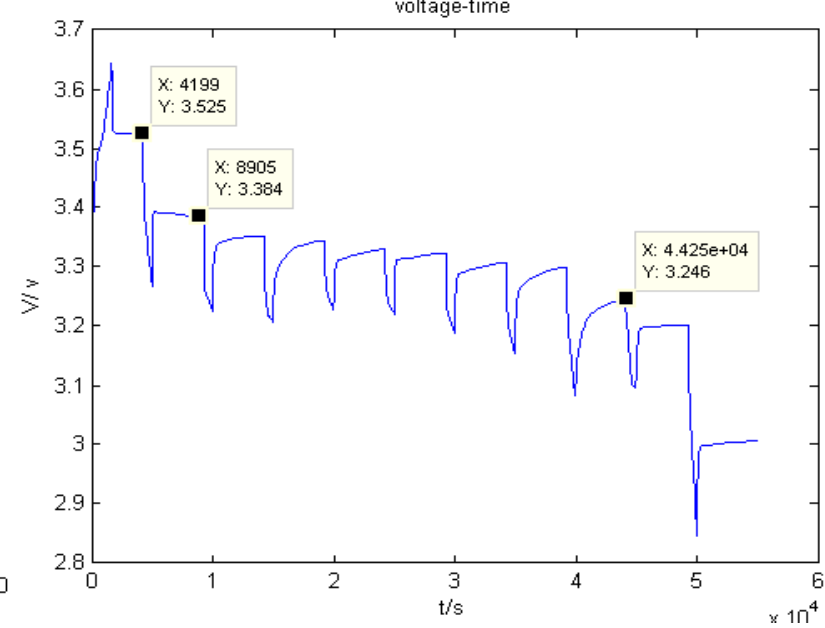

(b)

Figure 2 Charge and Discharge Characteristic Curves and voltage recovery test curve

After the needed parameters are set in the FIS toolbox, a surface for the FIS is accessible as shown in Figure. 3. It is direct and simple to view the relationship between SOC and the chosen parameters.

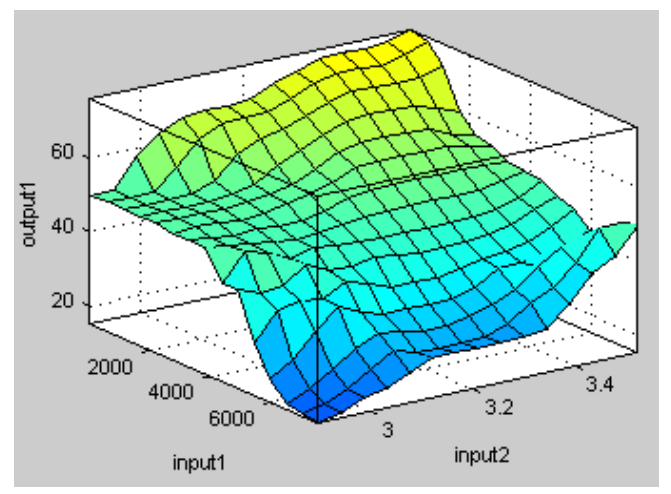

Figure 3 Surface for the FIS

\section{System verification and analysis}

In this section the results of the practiced methods are analyzed to see their efficiency in estimating battery SOC.

\subsection{The results of voltage recovery}

Figure.4shows the results of the voltage recovery test. Figure.4 (a) is the input current curve, Figure.4 (b) (c) (d) shows the voltage, SOC and battery temperature response. 


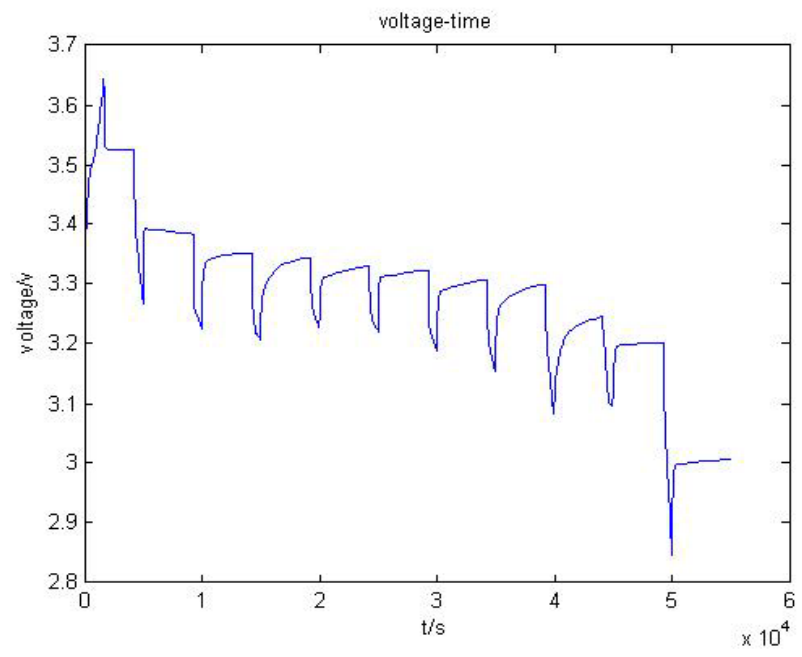

(a)

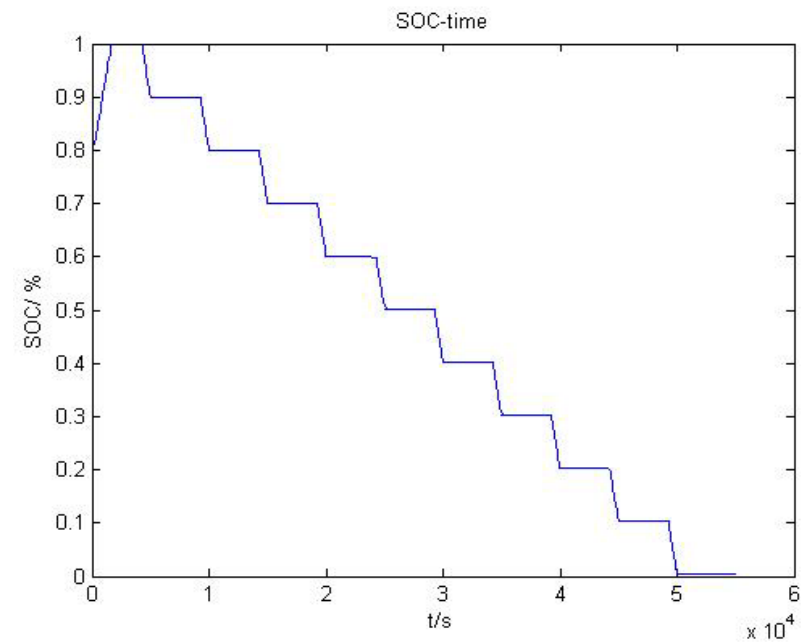

(c)

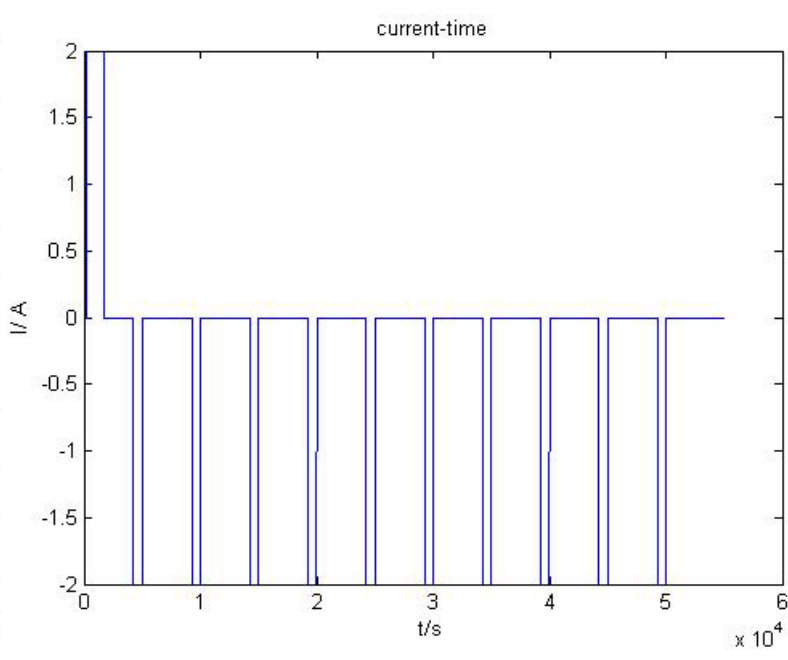

(b)

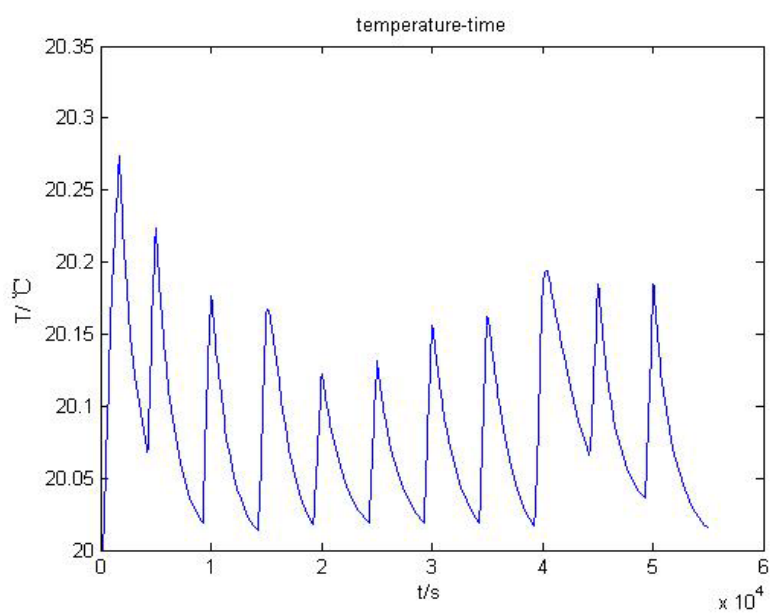

(d)

Figure 4 Results of the impulse discharge test

\subsection{The accuracy for FIS based on the discharge process}

The accuracy of FIS in predicting SOC was calculated by comparing the testing SOC to the real SOC datum. Generally speaking, the accuracy is higher when SOC is higher.

Table 2 Accuracy for FIS based on the discharge process

\begin{tabular}{|c|c|c|c|c|c|c|}
\hline No. & $\begin{array}{c}\text { True } \\
\text { SOC } \\
(\%)\end{array}$ & $\begin{array}{c}\text { Peak time } \\
(\mathrm{s})\end{array}$ & $\begin{array}{c}\text { Steady } \\
\text { voltage } \\
(\mathrm{v})\end{array}$ & $\begin{array}{c}\text { FIS result } \\
(\%)\end{array}$ & $\begin{array}{c}\text { Absolute Error Rate } \\
(\%)\end{array}$ & $\begin{array}{c}\text { Relative } \\
\text { Error Rate } \\
(\%)\end{array}$ \\
\hline 1 & 95 & 345 & 3.423 & 95.8 & 0.8 & 0.842 \\
\hline 2 & 85 & 1006 & 3.373 & 85.0 & 0 & 0 \\
\hline 3 & 75 & 1887 & 3.360 & 74.1 & 0.9 & 1.200 \\
\hline 4 & 65 & 2750 & 3.354 & 64.3 & 0.7 & 1.077 \\
\hline 5 & 55 & 3538 & 3.353 & 54.0 & 1.0 & 1.818 \\
\hline 6 & 45 & 4376 & 3.352 & 44.0 & 1.0 & 2.222 \\
\hline 7 & 35 & 5198 & 3.350 & 34.0 & 1.2 & 3.429 \\
\hline
\end{tabular}

\section{Conclusion}

In conclusion, this paper explored possible methods to make indication on the SOC and proposed 
a quick battery SOC test system based on FIS. Through battery discharge tests, the evaluation results for battery SOC can be obtained through the FIS block in Simulink.

\section{Acknowledgements}

The author would like to show great gratitude to Dr. Qing Wang for her supervision on this project and to the School of Engineering and Computing Sciences, Durham University for such a great experience to study there.

\section{References}

[1] Research, M., Environment, E., Power, E., Trends, E. and 2017-2027, L. (2017). Lithium-Ion Battery Market Report 2017-2027 - market research report. [Online] Reportlinker.com. Available at: http://www.reportlinker.com/p04771838/Lithium-Ion-Battery-Market-Report.html [Accessed 11 Jul. 2017].

[2] X. Wang, G. Gaustad, C. W. Babbitt, C. Bailey, M. J. Ganter, and B. J. Landi, "Economic and environmental characterization of an evolving Li-ion battery waste stream," Journal of environmental management, vol135, pp. 126-134, 2014.

[3] I. Buchmann, Batteries in a portable world. Richmond, B.C.: Cadex Electronics, 2001.

[4] Wikipedia. (2016). State of health. [Online] Available at: https://en.wikipedia.org/wiki/State_of_health [Accessed 21 May 2016].

[5] Singh, P. and Reisner, D. (n.d.). Fuzzy logic-based state-of-health determination of lead acid batteries. 24th Annual International Telecommunications Energy Conference.

[6] L. Zadeh, "Fuzzy sets", Information and Control, vol. 8, no. 3, pp. 338-353, 1965.

[7] L. Guo and G. Liu, "Research of Lithium-Ion Battery Sorting Method Based on Fuzzy C-Means Algorithm", AMR, vol. 354-355, pp. 983-988, 2011.

[8] P. Singh, R. Vinjamuri, X. Wang and D. Reisner, "Fuzzy logic modelling of EIS measurements on lithium-ion batteries", Electrochemical Acta, vol. 51, no. 8-9, pp. 1673-1679, 2006.

[9] P. Singh, C. Fennie and D. Reisner, "Fuzzy logic modelling of state-of-charge and available capacity of nickel/metal hydride batteries", Journal of Power Sources, vol. 136, no. 2, pp. 322-333, 2004.

[10] P. Singh, R. Vinjamuri, X. Wang and D. Reisner, "Design and implementation of a fuzzy logic-based state-of-charge meter for Li-ion batteries used in portable defibrillators", Journal of Power Sources, vol. 162, no. 2, pp. 829-836, 2006.

[11] A. Salkind, C. Fennie, P. Singh, T. Atwater and D. Reisner, "Determination of state-of-charge and state-of-health of batteries by fuzzy logic methodology", Journal of Power Sources, vol. 80, no. 1-2, pp. 293-300, 1999.

[12] H. Zhang, M.Y. Chow, “Comprehensive dynamic battery modelling for PHEV applications,” IEEE Power and Energy Society General Meeting, pp. 1-6, July 2010. 